\title{
DNA encoding individual mycobacterial antigens protects mice against tuberculosis
}

C.L. Silva,

V.L.D. Bonato and V.M.F. Lima
Departamento de Parasitologia, Microbiologia e Imunologia, Faculdade de Medicina de Ribeirão Preto, Universidade de São Paulo, Ribeirão Preto, SP, Brasil

\section{Correspondence \\ C.L. Silva \\ Departamento de Parasitologia, \\ Microbiologia e Imunologia \\ FMRP, USP \\ Av. Bandeirantes, 3900 \\ 14049-900 Ribeirão Preto, SP \\ Brasil \\ Fax: + 55-16-633-6631 \\ Email: clsilva@ fmrp.usp.br \\ Presented at the International \\ Symposium "The Third Revolution \\ on Vaccines: DNA Vaccines", \\ Belo Horizonte, MG, Brasil, \\ November 3-7, 1997. \\ Research supported by FAPESP CNPq and FINEP.}

Received O ctober 15, 1998 Accepted November 3, 1998

\begin{abstract}
Over the last few years, some of our experiments in which mycobacterial antigens were presented to the immune system as if they were viral antigens have had a significant impact on our understanding of protective immunity against tuberculosis. They have also markedly enhanced the prospects for new vaccines. We now know that individual mycobacterial protein antigens can confer protection equal to that from live BCG vaccine in mice. A critical determinant of the outcome of immunization appears to be the degree to which antigenspecific cytotoxic $\mathrm{T}$ cells are generated by the immune response. Our most recent studies indicate that DNA vaccination is an effective way to establish long-lasting cytotoxic $\mathrm{T}$ cell memory and protection against tuberculosis.
\end{abstract}

Key words - Tuberculosis

- DNA vaccination

- Cytokines

- Th1/Th2

\section{Introduction}

Tuberculosis kills 3 million people every year $(1,2)$. The disease is due to respiratory infection with Mycobacterium tuberculosis and the World Health Organization places its hope to bring tuberculosis under control on a combination of vaccination with bacillus Calmette-Guérin (BCG) to boost immunity and antibacterial drug treatment to directly kill the bacteria (1). Despite these efforts, there are still 10 million new cases worldwide every year, mainly in developing countries, and this changes little from year to year (2). The disease is now increasing again in the more affluent countries that have been so complacent about the situation elsewhere. Increasingly often the disease is caused by multi-drug resistant varieties of M. tuberculosis, and it is then not only highly infectious but also essentially incurable $(2,3)$. HIVinfected people are exquisitely susceptible and represent an increasing fraction of the population everywhere. Moreover, there are few signs of new antituberculosis drugs appearing in the near future, but there is real hope that recombinant DNA techniques will quickly produce major advances in the development of materials for vaccination.

The BCG vaccine is in widespread use against tuberculosis but has a doubtful impact on the global situation. It is a live vaccine derived from $M$. bovis by virulence attenuation during prolonged cultivation of the bacteria in the laboratory. In ten randomized controlled trials of BCG vaccines carried out since 1930, the protective efficacy against tuberculosis has ranged from 0 to $80 \%$ in different populations $(3,4)$. Thus, $\mathrm{BCG}$ is far from being an ideal vaccine 
against tuberculosis and in several countries it is virtually unused, not only because of doubts about its efficacy but also because it prevents the subsequent use of skin sensitivity tests to detect tuberculosis infection (3). Therefore, a new vaccine containing only a few key protective antigens could be the answer, but how are we to identify the protective mycobacterial antigens amongst the hundreds present in the whole microorganism? The classical approaches of separating bacterial components according to chemical, physical or immunological properties and testing the purified antigens have been disappointing because only modest protective effects have been obtained in animal models. Our answer was to take the cloned genes encoding some of the most immunologically prominent mycobacterial protein antigens and express them directly in antigen-presenting cells in mice $(5,6)$; to do this, we used DNA vaccination or a retroviral vector to transfect antigen-presenting cells.

\section{Immunization with a retroviral vector}

The initial steps in this process were taken when we used a retroviral shuttle vector to express the hsp65 antigen of Mycobacterium leprae in the monocyte-like tumor cell line J774 (BALB/c origin) (5). The cells presented the expressed antigen for specific recognition by $\mathrm{T}$ cells of the $\mathrm{CD} 4^{+}, \mathrm{CD} 8^{+}$ and $\gamma / \delta$ TCR types (7). When BALB/c mice were given a series of injections (ip or $i v$ ) of the transfected cells (J774-hsp65) they acquired a remarkably high degree of protection against a subsequent $i p$ or $i v$ challenge infection with virulent Mycobacterium tuberculosis H37Rv; bacterial numbers declined exponentially in internal organs and were, for example, 100-fold lower in livers after 5 weeks compared to control animals $(8,9)$. Injection of the protein with adjuvant was ineffective. Protection was dependent on tumor cell viability and was antigen specific; for example, there was no protection against challenge with Listeria monocytogenes or when $\mathrm{C} 57 \mathrm{Bl} / 6$ mice (haplotype d) were used $(6,8)$. The same retroviral construct was also used to transfect bone marrow cells in vitro and these cells were also able to generate specific protective immunity when they were used to reconstitute lethally gamma-irradiated mice (10). Evidently, features unique to tumor cells were not essential for the protective response and we investigated whether the virus-like endogenous origin of the antigen synthesized by the transfected cell was the key.

\section{The importance of a cytotoxic $T$ cell response}

The salient feature of endogenous antigen is presentation on MHC class I for recognition by $\mathrm{T}$ cells via $\mathrm{CD} 8$-associated receptors. As expected, limiting dilution analysis of $\mathrm{T}$ cells in the spleens of J774-hsp65 immunized mice showed that there was an increase in $\mathrm{CD}^{+} \mathrm{T}$ cells that was at least equal to the $\mathrm{CD}^{+}$response $(11,12)$ and very high frequencies were reached (1:250). The protein in the adjuvant, in contrast, selectively increased the frequency of $\mathrm{CD} 4^{+}$cells. Furthermore, $\mathrm{CD} 8^{+} \mathrm{T}$ cells cloned from the spleens of J774-hsp65-vaccinated mice were in general more effective than $\mathrm{CD} 4^{+}$cells in adoptive transfer of protective immunity to naive recipient $(9,12)$. The properties of 12 $\mathrm{CD} 4{ }^{+}$and $12 \mathrm{CD} 8^{+}$clones were compared. A spectrum of IFN- $\gamma$, IL-4 and specific cytotoxic activities was found among both types of clones. Supernatants from the clones were able to activate bone marrow-derived macrophages to inhibit intracellular M. tuberculosis $\mathrm{H} 37 \mathrm{Rv}$ in proportion to their IFN- $\gamma$ content. On the other hand, during direct cell:cell contact, clones that were cytotoxic showed antibacterial activity against the intracellular mycobacteria which was proportional to their cytotoxic activity. The two antimycobacterial effects, one of them IFN$\gamma$ dependent and the other cytotoxicity asso- 
ciated, were expressed independently. Upon adoptive transfer of clones into naive recipients, the most protective clones were found to be the most cytotoxic ones and their ability to produce IFN- $\gamma$ added little to the protective effect. The most cytotoxic clones were $\mathrm{CD} 8^{+}$clones (12).

\section{Immunization with plasmid DNA}

DNA vaccination is an alternative means of generating endogenous antigen and we reported the first evidence that this could also be used to generate protective anti-tuberculosis immunity at the first WHO DNA vaccine meeting in 1994 (6). We have obtained similar results with two different promoters, those for the CMV immediate early gene and murine hydroxymethylglutarylCoA-reductase, expressing a range of individual mycobacterial antigens following direct injection of plasmid DNA into muscle ( $2 \times 50 \mu \mathrm{g}$ on 3 or 4 occasions at 3-4-week intervals) (13). Effective genes were those encoding hsp65, hsp70, 36-kDa proline-rich antigen or ESAT- 6 and the degree of protection was equal to that obtained with live $\mathrm{BCG}$, depending on mouse strain and antigen (13). Analysis of the immune response to DNA expressing hsp65 showed that it was predominantly of the Th1 type (abundant IFN- $\gamma$, minimal IL-4) in ELISA and RT-PCR assays of tissues and antigen-stimulated $\mathrm{T}$ cells. Cytotoxic $\mathrm{CD} 8^{+} \mathrm{T}$ cells responded to peptides representing putative MHC class I restricted epitopes of hsp65 (13-15).

\section{Persistence of memory for specific cytotoxicity after DNA vaccination}

In an experiment comparing different means of immunizing with hsp65 we have now established that protocols that generate the antigen endogenously (J774-hsp65 or DNA vaccination) or that deliver the antigen into the cytosol (protein-loaded liposomes) generate protection and this is associated with a strong $\mathrm{CD} 8^{+}$response in which $\mathrm{CD} 44^{\mathrm{hi}}$ memory-associated cells are prominent (14). The greatest protection was seen 1 week after immunization with J774-hsp65 but this declined substantially by 8 months. Liposomes and DNA vaccination also gave substantial early protection equal to that of $\mathrm{BCG}$ and, whereas protection after liposomes declined like the protection after J774-hsp65, protection after hsp65-DNA was sustained like the BCG-induced protection. DNA vaccination also induced a sustained increase in the proportion of $\mathrm{CD} 8^{+}$splenocytes that were CD44hi. The frequencies of cytotoxic hsp65responsive cells among $\mathrm{CD} 44^{\mathrm{hi}} / \mathrm{CD}^{+}$ splenocytes at different intervals after the various vaccination protocols show that at 1 week after immunization the highest frequency was seen with J774-hsp65, reaching 1 in 8 , and this had substantially declined by 8 months after immunization. Hsp65-liposomes and hsp65-DNA also resulted in high frequency of CD44hi cells at 1 week (about 1 in 200) and this subsequently declined in the case of hsp65-liposomes but increased to 1 in 12 by 8 months in the case of DNA vaccination. This rising frequency of cytotoxic hsp65 specific $\mathrm{CD} 8^{+}$memory cells was also seen after BCG vaccination. One hypothesis to account for these findings is that only live BCG and DNA vaccination provide a persistent source of intracellular antigen and this is needed to sustain the cytotoxic $\mathrm{T}$ cell memory that is required for long lasting protection.

\section{Type 1 cytokines}

A striking difference between the immune response to DNA vaccination and the immune response to either BCG or M. tuberculosis infection is that DNA induces almost entirely a protective type 1 cytokine response, whereas the mycobacterial infections have a major component of non-cytotoxic $\mathrm{T}$ cells that produce a type 2 cytokine response (16). During infection or after immunization, CD4 
and CD8 hsp65-reactive $\mathrm{T}$ cells increased equally in spleens. During infection, the majority of these cells were CD44 ${ }^{\text {lo }}$ and produced IL-4 whereas after immunization the majority were CD44hi and produced IFN- $\gamma$. In adoptive transfer of protection to naive mice, the total CD8 cell population purified from spleens of immunized mice was more protective than that from infected mice. When the cells were separated into CD4 and CD8 types and then into CD44hi and CD44 $4^{\mathrm{lo}}$ types, CD44 ${ }^{\text {lo }}$ cells were found to be essentially unable to transfer protection, the most pro- tective CD44 $4^{\text {hi }}$ cells were CD8 and those from immunized mice were much more protective than those from infected mice. Thus, whereas the CD44 $4^{\text {lo }}$ IL-4-producing phenotype prevailed during infection, protection was associated with the CD8/CD44hi IFN- $\gamma$ producing phenotype that predominated after immunization. This conclusion was confirmed and extended by analysis of $16 \mathrm{hsp} 65$ reactive $\mathrm{T}$-cell clones from infected mice and 16 from immunized mice; the most protective clones, in addition, displayed antigen-specific cytotoxicity (16).

\section{References}

1. Kochi A (1991). The global tuberculosis situation and the new control strategies of the World Health Organization. Tubercle, 72: 1-6.

2. Snyder DE (Editor) (1994). Tuberculosis: the world situation. History of the disease and efforts to combat it. In: Tuberculosis. Back to the Future. Wiley, Chichester, 1333.

3. Fine PEM (1989). The BCG story: lessons from the past and implications for the future. Review of Infectious Diseases, 11: S353-S359.

4. Fine PEM (1988). BCG vaccination against tuberculosis and leprosy. British Medical Bulletin, 44: 704-716.

5. Silva CL, Palacios A, Colston MJ \& Lowrie DB (1992). Mycobacterium leprae 65hsp antigen expressed from a retroviral vector in a macrophage cell line is presented to $T$ cells in association with MHC class II in addition to MHC class I. Microbial Pathogenesis, 12: 27-38.

6. Lowrie DB, Tascon RE, Colston MJ \& Silva $C L$ (1994). Towards a DNA vaccine against tuberculosis. Vaccine, 12: 1537-1540.
7. Silva $C L$, Lukacs $K \&$ Lowrie DB (1993). Major histocompatibility complex non-restricted presentation to CD4 ${ }^{+}$T-lymphocytes of Mycobacterium leprae heatshock protein 65 antigen by macrophages transfected with the mycobacterial gene. Immunology, 78: 35-42.

8. Silva CL \& Lowrie DB (1994). A single mycobacterial protein (hsp65) expressed by a transgenic antigen-presenting cell vaccinates mice against tuberculosis. Immunology, 82: 244-248.

9. Silva $C L$, Silva MF, Pietro RCLR \& Lowrie DB (1994). Protection against tuberculosis by passive transfer with $T$ cell clones recognizing mycobacterial heat-shock protein 65. Immunology, 83: 341-346.

10. Silva $C L$, Pietro RCLR, J anuário $A$, Bonato VDL, Lima VMF, Silva MF \& Lowrie DB (1995). Protection against tuberculosis by bone marrow cells expressing mycobacterial hsp65. Immunology, 86: 519-524.

11. Lowrie DB, Tascon RE \& Silva CL (1995). Vaccination against tuberculosis. International Archives of Allergy and Immunology, 108: 309-312.
12. Silva $C L$, Silva $M F$, Pietro RCLR \& Lowrie DB (1996). Characterization of the T cells that confer a high degree of protective immunity against tuberculosis in mice after vaccination with tumor cells expressing mycobacterial hsp65. Infection and Immunity, 64: 2400-2407.

13. Lowrie DB, Silva CL, Colston MJ, Ragno $S \&$ Tascon RE (1997). Protection against tuberculosis by plasmid DNA. Vaccine, 15: 834-838.

14. Lowrie DB, Silva CL \& Tascon RE (1997). Genetic vaccination against tuberculosis. Springer Seminars in Immunopathology, 19: 161-173.

15. Lowrie DB, Silva CL \& Tascon RE (1997) DNA vaccines against tuberculosis. Immunology and Cell Biology, 75: 591-594.

16. Bonato VLD, Lima VMF, Tascon RE, Lowrie DB \& Silva CL (1998). Identification and characterization of protective $\mathrm{T}$ cells in hsp65 DNA vaccinated and Mycobacterium tuberculosis infected mice. Infection and Immunity, 66: 169-175. 